\title{
ON REGULAR FAMILIES OF CURVES ${ }^{1}$
}

\section{HASSLER WHITNEY}

A family $F$ of non-intersecting curves filling a metric space is called regular if, in a neighborhood of any point $p$, it is homeomorphic with a family of straight lines. We have given in another paper ${ }^{2}$ a necessary and sufficient condition, which we shall call $\left(\mathrm{A}^{\prime}\right)$ (to be described below), that a family $F$ be regular. We shall prove in this note that the following condition is sufficient:

(A) Given any point $p$, and a direction on the curve through $p$, there is an arc $p q$ in this direction with the following property. For every $\epsilon>0$ there is a $\delta>0$ such that for any $p^{\prime}$, with $\rho\left(p^{\prime}, p\right)<\delta$, there is an arc $p^{\prime} q^{\prime}$ of $C\left(p^{\prime}\right)$ such that

$$
p^{\prime} q^{\prime} \subset V_{\epsilon}(p q), \quad q^{\prime} \subset V_{\epsilon}(q) .
$$

The condition $\left(\mathrm{A}^{\prime}\right)$ is the same, except that after (1), we add:

(2) If $r^{\prime}$ and $s^{\prime}$ are on $p^{\prime} q^{\prime}$ and $\rho\left(r^{\prime}, s^{\prime}\right)<\delta$, then $\delta\left(r^{\prime} s^{\prime}\right)<\epsilon$.

From the present theorem it is clear that the families of curves recently defined by $\mathrm{Niemitzki}^{3}$ are regular.

To prove the theorem, suppose $(\mathrm{A})$ holds, but $\left(\mathrm{A}^{\prime}\right)$ does not. Then the following is true:

(B) There is a point $p$, and a direction of the curve $C(p)$, such that for any arc $p q$ on $C(p)$ in this direction, there is an $\epsilon>0$, such that for any $\delta>0$, there is a point $p^{\prime}$, with $\rho\left(p^{\prime}, p\right)<\delta$, such that for any $q^{\prime}$ on $C\left(p^{\prime}\right)$,

(3) either $p^{\prime} q^{\prime} \mp V_{\epsilon}(p q)$, or $q^{\prime} \mp V_{\epsilon}(q)$,

1 Presented to the Society, April 27, 1940.

2 Annals of Mathematics, (2), vol. 34 (1933), pp. 244-270. We refer to this paper as RF. By RF, Theorem $7 \mathrm{~A}, F$ is regular as there defined. The converse is proved as follows. By Theorem 17A, there is a cross-section $S$ through $p$. In a neighborhood of $p$, the curves are orientable (this is easily seen, for instance, with the help of Theorem 9B). Choose an open subset $S^{\prime}$ of $S$, and let $U$ be all points $q^{\prime}=g^{\prime}(q, \alpha), q$ in $S^{\prime},|\alpha|<\epsilon$ (see RF, §15); $U$ is a neighborhood of $p$, expressed as the product of $S^{\prime}$ and the open line segment $-\epsilon<\alpha<\epsilon$.

By a curve, we shall mean here the topological image of an open line segment or of a circle. We shall use $\rho(p, q)$ for distance, $\delta(A)$ for the diameter of the set $A$, and $V_{\epsilon}(A)$ for the set of all points $p, \rho(p, A)<\epsilon$. Let $C(p)$ mean the curve of $F$ through $p$.

${ }^{3}$ V. Niemytzki, Recueil Mathématique de Moscou, vol. 6 (48) (1939), pp. 283-292. We mention two further papers in the subject: H. Whitney, Duke Mathematical Journal, vol. 4 (1938), pp. 222-226, showing that if the curves fill a region in 3-space, a cross-section may be chosen so as to be a 2-cell; W. Kaplan, Duke Mathematical Journal, vol. 7 (1940), pp. 154-185, studying families filling the plane. 
(4) or there are points $r^{\prime}, s^{\prime}$ on $p^{\prime} q^{\prime}$ such that $\rho\left(r^{\prime}, s^{\prime}\right)<\delta$, and $\delta\left(r^{\prime} s^{\prime}\right) \geqq \epsilon$.

Choose a point $p$ and a direction on $C=C(p)$, by (B). Choose $q$ on $C$ in this direction, by (A). Choose $\epsilon>0$ by (B). For each positive integer $i$, choose $\delta_{i}$ by (A), with $\epsilon$ replaced by $\epsilon / i$. Choose $p_{i}$ by (B), with $\delta$ replaced by $\delta_{i}$. Choose $q_{i}$ by $(\mathrm{A})$, with $p^{\prime}$ replaced by $p_{i}$; then

$$
p_{i} q_{i} \subset V_{\epsilon / i}(p q), \quad q_{i} \subset V_{\epsilon / i}(q) .
$$

By $(\mathrm{B})$, as $\epsilon_{i}<\epsilon$, we may choose $p_{i}^{\prime}$ and $q_{i}^{\prime}$ on $p_{i} q_{i}$ so that

$$
\rho\left(p_{i}^{\prime}, q_{i}^{\prime}\right)<\delta_{i}, \quad \delta\left(p_{i}^{\prime} q_{i}^{\prime}\right) \geqq \epsilon .
$$

By (6), we may choose $r_{i}$ on $p_{i}^{\prime} q_{i}^{\prime}$ so that $\rho\left(p_{i}^{\prime}, r_{i}\right) \geqq \epsilon / 2$. By (5) and (6), we may choose a subsequence so that for some points $p^{\prime}$ and $r$ on $p q$,

$$
p_{\lambda_{i}}^{\prime} \rightarrow p^{\prime}, \quad q_{\lambda_{i}}^{\prime} \rightarrow p^{\prime}, \quad r_{\lambda_{i}} \rightarrow r
$$

then $r \neq p^{\prime}$. Say, for definiteness, that $r$ is in the direction of $q$ from $p^{\prime}$. The set of such points $r$ which are limits of such sequences $\left\{r_{\lambda_{i}}\right\}$ forms a closed set, which, by (5), is in $p^{\prime} q$; we shall let $r$ be the point furthest from $p^{\prime}$. (It might be $q$.)

Assuming that (A) holds for the point $r$ and the direction away from $p^{\prime}$, we shall arrive at a contradiction. Choose a point $s$ on $C$ in this direction from $r$, by (A). (If $C$ is a closed curve, it might happen that $s$ is on the arc pr.) Choose $r^{\prime}$ and $s^{\prime}$ on $C$ just behind and just in front of $r$, so that $r^{\prime}$ is on neither $p p^{\prime}$ nor $r s$, and $s^{\prime}$ is not on $p^{\prime} r$. We shall show that for any $\epsilon^{\prime}>0$ there is an integer $j$ and a point $s_{j}$ on $p_{j}^{\prime} q_{j}^{\prime}$ within $\epsilon^{\prime}$ of $s^{\prime}$; as $s$ is in $p q$, by (5), this will contradict the definition of $r$, and thus prove the theorem.

Set

$$
4 \eta=\min \left[\rho\left(r^{\prime}, r s\right), 2 \epsilon^{\prime}\right] .
$$

Choose $r_{-}, r_{+}, s_{-}, s_{+}$on $C$ in the order $r_{-} r^{\prime} r_{+} r s_{-} s^{\prime} s_{+} s$, so that $r_{-}$is not in $r s$ and $s_{+}$is not in $p r$ (if $C$ is closed), and so that

$$
r_{-} r^{\prime} r_{+} \subset V_{\eta}\left(r^{\prime}\right), \quad s_{-} s^{\prime} s_{+} \subset V_{\eta}\left(s^{\prime}\right)
$$

Set

$$
2 \epsilon^{\prime \prime}=\min \left[\rho\left(p r_{-}, r_{+} q\right), \rho\left(r s_{-}, s_{+} s\right), \eta\right] .
$$

Using $r, s$, and $\epsilon^{\prime \prime}$, choose $\delta^{\prime \prime}>0$ by (A). By (7), we may choose $j$ so that

$$
\epsilon / j<\epsilon^{\prime \prime}, \rho\left(p_{j}^{\prime}, p^{\prime}\right)<\epsilon^{\prime \prime}, \rho\left(q_{j}^{\prime}, p^{\prime}\right)<\epsilon^{\prime \prime}, \rho\left(r_{j}, r\right)<\delta^{\prime \prime} .
$$


By the choice of $\delta^{\prime \prime}$, we may choose $s^{*}$ on $C\left(r_{j}\right)$ so that

$$
r_{j} s^{*} \subset V_{\epsilon^{\prime \prime}}(r s), \quad s^{*} \subset V_{\epsilon^{\prime \prime}}(s) \text {. }
$$

As $r_{j} s^{*}$ is a connected set, (11), (12) and (10) show that there is a point $s_{j}$ on it such that $\rho\left(s_{j}, r s_{-}+s_{+} s\right) \geqq \epsilon^{\prime \prime}$; hence, by $(12), \rho\left(s_{j}, s_{-} s_{+}\right)<\epsilon^{\prime \prime}$, and by (9),

$$
\rho\left(s_{j}, s^{\prime}\right)<\epsilon^{\prime \prime}+\eta<2 \eta \leqq \epsilon^{\prime} .
$$

By (12) and (8),

$$
\rho\left(r^{\prime}, r_{j} s_{j}\right)>2 \eta
$$

By (5) and (11),

$$
p_{j}^{\prime} q_{j}^{\prime} \subset V_{\epsilon^{\prime \prime}}(p q) .
$$

By (11), (10), (5) and (9), there are points $p_{j}^{*}$ in $p_{j}^{\prime} r_{j}$ and $q_{j}^{*}$ in $r_{j} q_{j}^{\prime}$ such that

$$
\rho\left(p_{j}^{*}, r^{\prime}\right)<2 \eta, \quad \rho\left(q_{j}^{*}, r^{\prime}\right)<2 \eta .
$$

By this and (14), the arc $r_{j} s_{j}$ is contained in the $\operatorname{arc} p_{j}{ }^{*} r_{j} q_{j}{ }^{*} \subset p_{j}^{\prime} q_{j}^{\prime}$. Hence $s_{j} \subset p_{j}^{\prime} q_{j}^{\prime}$, which, with (13), gives the contradiction. 